\title{
La Pontificia Universidad de San Javier: Agente de transformación de la sociedad panameña
}

\author{
Cid Labra, Patricia L. \\ Universidad Católica Santa María la Antigua \\ Centro de Investigaciones para la Conservación del Patrimonio Arquitectónico y Urbano \\ Panamá, Rep. de Panamá \\ pcidl@usma.com.pa; ORCID 0000-0002-3139-7245 \\ Casini, Leonardo \\ Universidad Católica Santa María la Antigua \\ Centro de Investigaciones para la Conservación del Patrimonio Arquitectónico y Urbano \\ Panamá, Rep. de Panamá \\ centroinvest.copau@gmail.com; ORCID 0000-0002-5272-3038
}

\section{Abstract}

The Royal and Pontifical University of San Javier -the first university of Panamá- represents one of the most important legacies of the religious order in the country where, through the labor of Ignatian model, was a key piece in the transformation of the society in the 18th century from a religious, service and educational basis. With the interest of valuing the culture that comprises the monumental asset and the impact that the order had, it was sought to inquire into the presence of its main representatives between 1737 and 1767 and their contribution to the university educational level. The research was humanistic and heuristic, hermeneutical, and deductive-inductive methods were used. Additional information was obtained that allowed to reconstruct of the intramural activities of the Xavier University, as well as, the recognition of its teachers in the expulsion trips of the Viceroyalty of Peru and its final location in Italian lands. The study route included some of the main European reference centers such as the Archivum Romanum Societatis lesu in Rome, the Archivo de España de la Compañía de Jesús en Alcalá de Henares, the Archivo General de Indias, and others. The Jesuit project was ambitious for the region and designed to compete with the Universities of Bogota, Quito, and Lima, the three prestigious references in South America. The product of the spiritual, intellectual, and moral influence of the Society of Jesus in the socio-cultural formation in Panama has been incalculable.

Keywords: History of America, Society of Jesus, history of church, social and cultural history, cultural heritage.

\section{Resumen}


La Real y Pontificia Universidad de San Javier -primera universidad de Panamá- representa uno de los legados más importantes de las órdenes religiosas en el territorio donde, a través de la labor de impronta ignaciana, fue pieza clave en la transformación de la sociedad del siglo XVIII desde una base religiosa, de servicio y de instrucción. Con el interés de valorizar la cultura que comprende el bien monumental y el impacto que tuvo la orden, se buscó indagar en la presencia de sus principales representantes entre 1737 y 1767 y su aporte a la educación superior. La investigación fue de tipo humanística y se emplearon los métodos heurístico, hermenéutico y deductivo-inductivo. Se obtuvo además, información adicional que permitió reconstruir la tarea intramuros de la Universidad Javeriana, así como el reconocimiento de sus docentes en los viajes de expulsión del Virreinato del Perú y su localización final en tierras italianas. La ruta de estudio incluyó algunos de los principales centros de referencia europeos como el Archivum Romanum Societatis lesu en Roma, el Archivo de España de la Compañía de Jesús en Alcalá de Henares, el Archivo General de Indias, entre otros. El proyecto de los Jesuitas fue ambicioso para la región y pensado para competir con las Universidades de Bogotá, Quito y Lima, las tres prestigiosas referencias en Sudamérica. El fruto de la influencia espiritual, intelectual y moral de la Compañía de Jesús en la formación socio-cultural en Panamá ha sido incalculable.

Palabras claves: Historia de América, Compañía de Jesús, historia de la iglesia, historia social y cultural, patrimonio cultural.

\section{INTRODUCCIÓN}

La Real y Pontificia Universidad de San Javier es la obra cumbre del aporte cultural de la Compañía de Jesús en Panamá y su conjunto monumental simboliza el final de la etapa colonial de la historia de los hijos de Loyola en estas tierras. Sus instalaciones reúnen el testimonio de las actividades, misiones y enseñanzas que la orden religiosa desde aquí promovió como centro de gobierno en el istmo, y de referencia cultural y de instrucción para la sociedad del siglo XVIII [1].

El presente trabajo $1^{*}$ se concentró en analizar y comprender los aspectos socio-culturales y académicos comprendidos en el complejo conventual jesuita entre 1737 y 1767, desde la construcción después del fuego grande hasta el momento de expulsión de los religiosos del territorio.

En la actualidad, el patrimonio documental de los jesuitas de Panamá se encuentra * Los resultados que aquí se exponen pertenecen al proyecto "La Real y Pontificia Universidad de San Javier. La educación universitaria y la arquitectura conventual de la Compañía de Jesús en Panamá durante el siglo XVIII”, financiado por la Universidad Católica Santa María la Antigua, en el 2019 - 2020. 
distribuido en diversos archivos religiosos, estatales y en bibliotecas de Europa y América. Por esta razón, la ruta de estudio de esta investigación solo incluyó algunos de los principales centros de referencia europeos como el Archivum Romanum Societatis lesu (ARSI) en Roma, el Archivo de España de la Compañía de Jesús en Alcalá de Henares (AESI-A), el Archivo General de Indias (AGI) en Sevilla, y archivos estatales y bibliotecas en Madrid y Panamá.

\section{MÉTODO}

La investigación histórica de tipo cualitativa fue dirigida al estudio de un caso del patrimonio arquitectónico nacional. Se buscó analizar histórica, humanística y culturalmente el aporte de la Compañía de Jesús en la sociedad de Panamá del siglo XVIII y en la educación universitaria. Fue desarrollado en 2 fases: preparatoria y analítica.

\section{A. Fase Preparatoria}

Se utilizó el método heurístico y comportó la consultación de documentos históricos (cartas, comunicaciones, diarios de memorias, ilustraciones, cartografías) en archivos jesuitas y estatales en Roma, Alcalá de Henares, Madrid y Sevilla, así como, fuentes históricas literarias en la Biblioteca Nacional de Madrid y Panamá, y publicaciones de reconocidos autores locales e internacionales en los temas de la sociedad colonial panameña, la historia de la iglesia y la labor de la orden en Panamá.

\section{B. Fase Analítica}

Los métodos empleados fueron el hermenéutico para el análisis de las fuentes, verificación de datos por comparación y producción del conocimiento, y el deductivo - inductivo para la síntesis y reconstrucción de los hechos históricos. Como sub-método se recurrió al uso de la cronología para la organización secuencial de los eventos.

\section{RESULTADOS}

Debido a lo extenso de los resultados por comunicar, en esta ocasión solo presentaremos algunos de los datos más relevantes de nuestra investigación y que consideramos podrán brindar nuevas luces al estudio de los jesuitas en Panamá.

\section{A. Principales representantes de la Universidad de San Javier (1737 - 1767)}

A continuación, presentamos los nombres de las principales figuras comprometidas en impartir un alto nivel de educación superior, que fuera accesible también para los interesados en América Meridional [2]. Sus conocimientos, formación religiosa y cultura personal influyeron en las decisiones de la orden, en la instrucción, y en la sociedad capitalina y las comunidades del interior del país. 
Tabla 1. Rectores del Colegio de Panamá y Universidad de San Javier (USJ)

\begin{tabular}{|c|c|c|c|}
\hline Periodo & Nombre & Nacionalidad & Cargos y Docencia \\
\hline $1737-1740$ & Ignacio Cayroni (Ignazio Caironi) & Italiano & Rector del Colegio \\
\hline $1740-1743$ & $\dot{¿} ?$ & & \\
\hline $1744-1747$ & Pablo Maroni (Paolo Maroni) & Italiano & Rector del Colegio \\
\hline $1747-1750$ & Lucas Portulani (Luca Portolani) & Italiano & $\begin{array}{c}\text { Rector del Colegio y primer Rector de } \\
\text { la USJ, Prof. de Gramática }\end{array}$ \\
\hline 1751 & José Fiñón & & Rector del Colegio y la USJ \\
\hline $1751-1753$ & Jerónimo de Herce (o de Herze) & & Rector del Colegio y la USJ \\
\hline $1753-1757$ & ¿? & & \\
\hline $1757-1759$ & Juan Antonio Giraldo & Panameño & Rector del Colegio y la USJ \\
\hline $1760-1763$ & Bernardo Recio & Español & $\begin{array}{l}\text { Rector del Colegio y la USJ, } \\
\text { Teología y Moral }\end{array}$ \\
\hline$* 1767$ & Francisco Pallares & Ecuatoriano & $\begin{array}{l}\text { Rector del Colegio y la USJ, Catedrático de } \\
\text { Filosofía y Teología Escolástica }\end{array}$ \\
\hline
\end{tabular}

El esplendor arquitectónico del antiguo Collegium Panamense cum Universitate se lo debemos, principalmente a dos personas que determinaron la caracterización constructiva y estética del complejo, convirtiéndolo en una de las principales referencias de la urbe. El primero, el jesuita italiano Ignacio María Cayroni, quien inicia y finaliza las estructuras del convento después del incendio grande (1737-1739). Y el segundo, el padre español Bernardo Recio, quien entre 1760 y 1763 transporta las piedras de los muros del edificio eclesiástico de Panamá la Vieja (Andava egli molte volte in persona al porto, e alla spiaggia, e caricava sulle sue spalle quel che poteva, e per le pubbliche strade della Città, con sudore e fatica lo portava al Collegio) $2^{* *}$ y junto con ladrillos y argamasa realiza la nueva Iglesia de La Compañía, que luego quedará inconclusa ante la partida de los jesuitas en 1767 [3]. Años antes, en cambio, en 1744, el P. Pablo Maroni inicia las clases en la futura universidad, gracias a la autorización de la Real Audiencia de Panamá y los donativos del presbítero Francisco Javier de Luna Victoria, quien será Obispo de Tierra Firme en 1751. Seis años más tarde, y casi al finalizar su trienio, el P. Lucas Portulani -enseñante de gramática- portará el gobierno como primer rector oficial de la Universidad Javeriana [4].

El único panameño que encontramos, ejerció en el último período de los años 50 . Es el P. Juan Antonio Giraldo, quien una década antes había sido enseñante de Filosofía en la Universidad de San Gregorio Magno, en Quito. Mientras que, el P. Francisco Pallares concluirá la experiencia de la historia colonial de los jesuitas en Panamá, luego de dictada la orden de expulsión por Carlos III en 1767 [5].

\section{B. Los Jesuitas de Panamá en la Provincia de Quito (1690 - 1775)}

\footnotetext{
** Muchas veces, iba él mismo al puerto y a la playa, y cargaba sobre sus hombros lo que podía. Y por las calles públicas de la ciudad, con sudor y cansancio, lo llevaba al Colegio. (traducción de los autores)
} 
El siguiente análisis está basado en los Catálogos Triennales y en el Catalogum personarum et officiorum Provinciae Quitensis Societatis Jesu del ARSI, y cuantifica la presencia de la orden de la Compañía de Jesús en la Provincia de Quito, a la cual Panamá fue destinada. Los dos colores, amarillo y verde, corresponden al momento de división organizativa del territorio americano y creación de la Provincia Novi Regni et Quitensis en 1696.

La máxima participación de la orden en la Provinciae Quitensis en el Virreinato Peruano fue registrada entre 1761 y 1767, éste último, el año de expulsión de los jesuitas. Posterior a esto, el gráfico ilustra la paulatina disminución por muerte natural en sus periodos de extradición en Italia.

En cuanto a Panamá, el período analizado (en rojo) refleja los años de los jesuitas en Panamá la Nueva -actual Casco Antiguo de la ciudad de Panamá- y en sus misiones en el interior del istmo.

Tabla 2. Los Jesuitas de Panamá en la Provincia de Quito

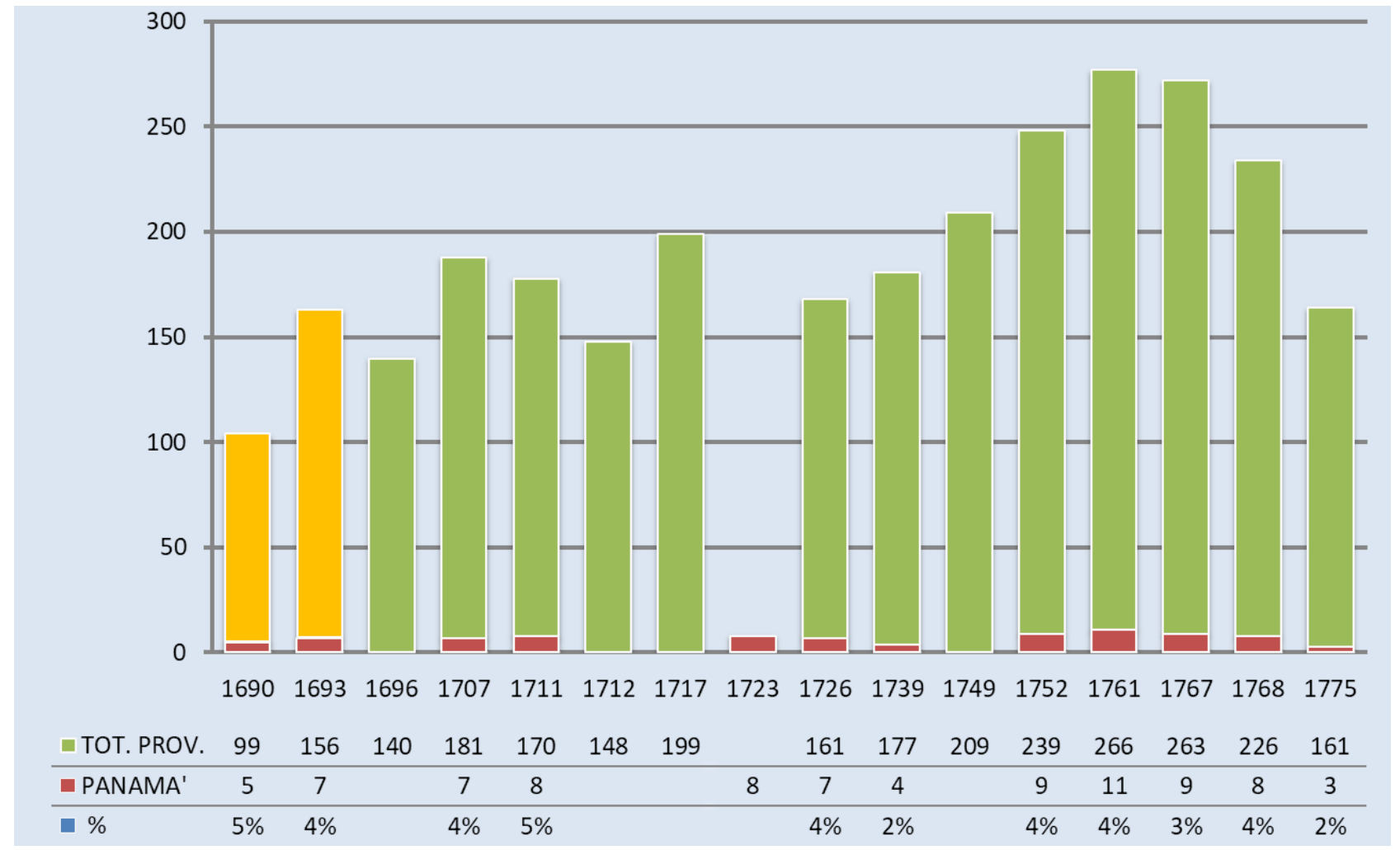

Por lo general, el 4\% de los padres se mantuvo destinado a la Plaza de Panamá; mientras que, los momentos más críticos se presentaron en tres ocasiones. La primera, al terminar el complejo del nuevo Colegio en 1739, donde se enviaron a 4 jesuitas -bajo la dirección del P. Cayroni- para comenzar las clases después del incendio grande.

La segunda, diez años más tarde sin registro de los religiosos. Esto se debió, probablemente, a la suspensión de clases que ocurrió por varios meses entre 1747 y 1748 . La orden provino 
del Padre Viceprovincial Guillermo Grebmer ante la poca cantidad de matrículas y el supuesto desinterés en las materias que se dictaban, describiendo la actitud de los seglares con el proverbio de saecularibus non datur scientia [4]. Aun así, el 3 de junio de 1749 se oficializó la fundación de la Real y Pontificia Universidad de San Javier por Real Cédula de Fernando VI [6].

Hemos encontrado en el Status Temporalis Povinciae Quitensis Anno 1752 del ARSI [7], que para este año, se registró la presencia de 9 jesuitas en Panamá ( 6 sacerdotes y 3 coadjutores). De estos, 4 sacerdotes eran enseñantes y cada uno fue destinado a un curso de Filosofía, Gramática, Teología Escolástica y Teología Moral.

La tercera ocasión, la hallamos en el último censo de 1775, luego del decreto de supresión del Papa Clemente XIV dictado dos años antes. Para este entonces, se registraron 3 jesuitas de Panamá en extradición en Italia.

\section{La extradición a tierras italianas (1767 - 1775)}

En la Biblioteca Nacional de España, en Madrid, se conserva el diario contable de los pagos vitalicios de los jesuitas americanos que desembarcaron en el Puerto de Santa María, en su viaje de extradición hacia Italia [8]. Con respecto al Colegio de Panamá, encontramos registrada la partida de 9 jesuitas desde Portobelo, de los cuales llegan al puerto: 7 sacerdotes y 2 coadjutores. De estos, 3 fallecen en el viaje.

En el cuarto trimestre de 1775, los registros indican que permanecen con vida solo 3 de los 9 que partieron inicialmente. Están ubicados en Italia, en las ciudades de Rimini y Ravenna: Francisco Pallares (último rector), Ignacio Peramás y José Archs.

\section{CONCLUSIONES}

La Real y Pontificia Universidad de San Javier fue la primera universidad jesuita de Tierra Firme y uno de los complejos conventuales más pequeños de la Provinciae Quitensis, pero de imponente presencia en la Panamá colonial amurallada [9]. Debido a las problemáticas de transporte, las rutas intercontinentales y la orografía del territorio, la Universidad Javeriana de Panamá brindó a las comunidades de Centroamérica la posibilidad de obtener un nivel de educación superior en la región, evitando tener que movilizarse a las accidentadas zonas montañosas de Santa Fe de Bogotá y Quito, o realizar una larga travesía marítima para llegar a Lima.

La importante labor de la Compañía de Jesús en el cultivo del intelecto y del espíritu impactó a los estratos más humildes y a los más elevados, participando como agente de transformación a través de la educación integral del individuo. Los 30 años de existencia del Collegium Panamense cum Universitate, los eventos y actuaciones que la orden aquí condujo forman parte de la historia de la iglesia, de la sociedad panameña y de la historia de América. El legado cultural y de conocimiento que ofrecieron los misioneros jesuitas y 
sus principales representantes, hoy lo vemos simbolizado en el patrimonio arquitectónico y religioso de su complejo conventual universitario y sus memorias.

\section{Referencias}

[1] P.Cid and B. Cardoze, "Historia constructiva del Complejo Monástico de la Compañía de Jesús en el Casco Antiguo de Panamá y análisis de su tutela", Investigacion y Pensamiento Critico, vol. 6, n², Panamá: Universidad Católica Santa María la Antigua, 2018, pp. 30-51.

[2] J. M. Sariego, "Evangelizar y educar. Los jesuitas de la Centroamérica colonial", Estudios Centroamericanos, vol. 65, n723, El Salvador: Universidad Centroamericana "José Simeón Cañas", 2010, pp. 11-24.

[3] G. Janer, "Vita del Sacerdote D. Bernardo Recio", Foligno: Giovanni Tommassini Stampatore Vescovile, 1794, pp. 149-150.

[4] J. Jouanen, "Historia de la Compañía de Jesús en la antigua Provincia de Quito. 1570-1773", tomo II, Quito: Editorial Ecuatoriana, 1943, 235-241.

[5] A. Morin Couture, "Apuntes de Historia de la Iglesia de Panamá. Período Colonial", tomo la, Panamá: Ministerio de Educación/Instituto Nacional de Cultura, 2008, pp. 201-215, 314-323.

[6] M. Cambra, "Los jesuitas y sus obras en Panamá. En todo, amar y servir", Panamá: Editorial La Antigua, 2013, pp. 29-37.

[7] ARSI, Novi Regni et Quitensis 11, Cat. Trien. Quit. 1690-1756.

[8] BNE, "Estado general en que se demuestra el número y clase de regulares de la extinguida religión de la Compañía [de Jesús] que existían en España cuando se les intimó el Real Decreto de expulsión: los que han llegado de los reinos de la América al Puerto de Santa María, los que han fallecido desde aquella época hasta [el] 31 de octubre de 1775", MSS/9136

[9] M. Cicala, "Descripción histórica de la Provincia de Quito de la Compañía de Jesús", Quito: Instituto Geográfico Militar, 1994, pp. 240.

\section{Autorización y Licencia CC}

Los autores autorizan a APANAC XVIII a publicar el artículo en las actas de la conferencia en Acceso Abierto (Open Access) en diversos formatos digitales (PDF, HTML, EPUB) e integrarlos en diversas plataformas online como repositorios y bases de datos bajo la licencia CC: Attribution-NonCommercial-ShareAlike 4.0 International (CC BY-NC-SA 4.0) https://creativecommons. org/licenses/by-nc-sa/4.0/.

Ni APANAC XVIII ni los editores son responsables ni del contenido ni de las implicaciones de lo expresado en el artículo. 